\title{
Publisher's Note: Loading-unloading hysteresis loop of randomly rough adhesive contacts [Phys. Rev. E 92, 062404 (2015)]
}

\author{
Giuseppe Carbone, Elena Pierro, and Giuseppina Recchia
}

(Received 20 April 2016; published 27 April 2016)

DOI: 10.1103/PhysRevE.93.049908

This paper was published online on 8 December 2015 with errors in Figs. 6 and 7 and their captions. Figures 6 and 7 have been replaced and their captions corrected as of 20 April 2016. The figures and their captions are incorrect in the printed version of the journal; therefore for the benefit of the print readership, the figures and their captions are replicated below.
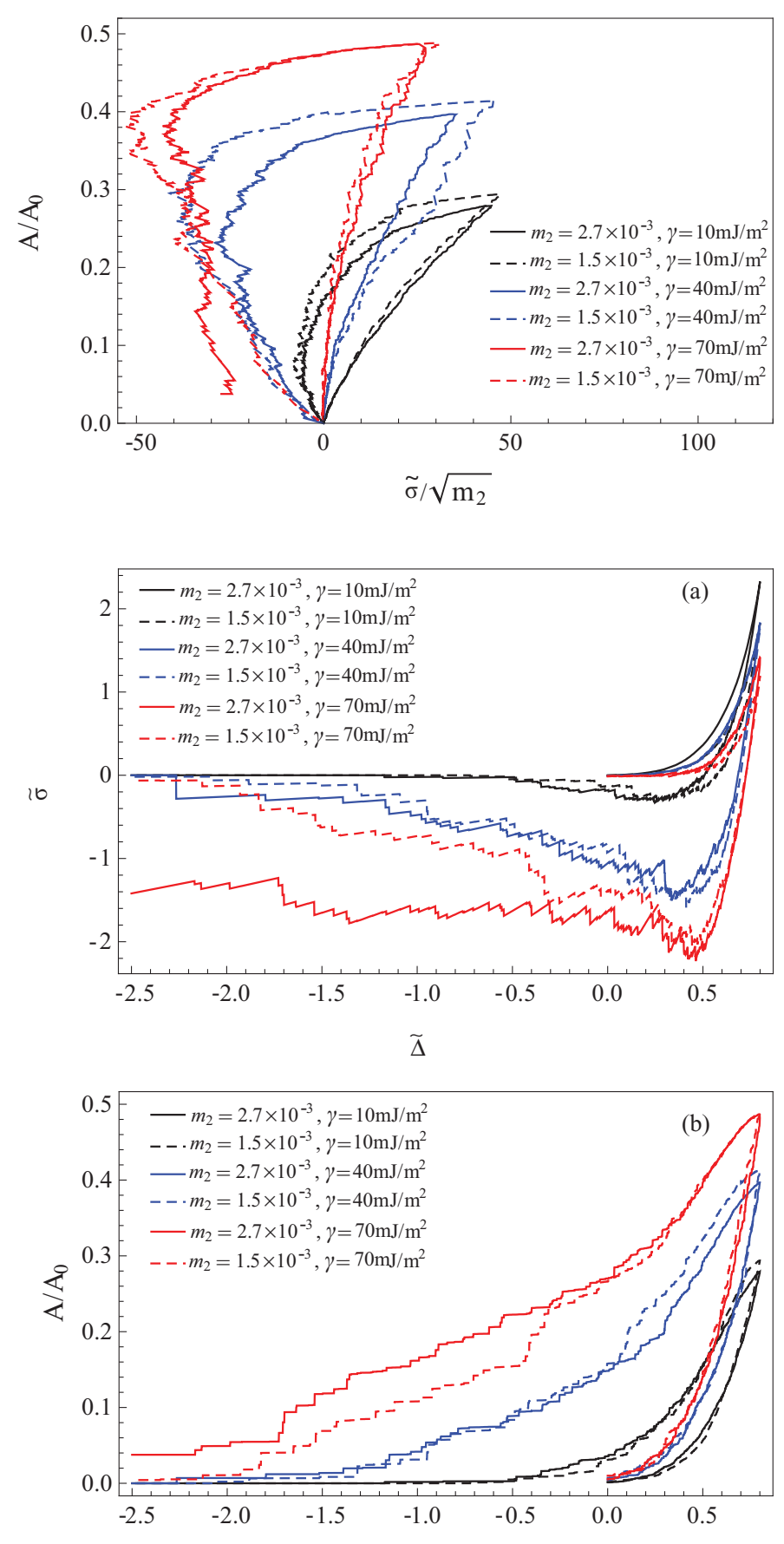

$\widetilde{\Delta}$
FIG. 6. The true contact area $A / A_{0}$ as a function of the dimensionless quantity $m_{2}^{-1 / 2} \tilde{\sigma}$ for two different Hurst exponents $H=0.8\left(m_{2}=2.7 \times 10^{-3}\right), H=0.9\left(m_{2}=1.5 \times 10^{-3}\right)$, and for three different values of energy of adhesion, $\gamma=$ $0.01 \mathrm{~J} / \mathrm{m}^{2}$ (black curves), $\gamma=0.04 \mathrm{~J} / \mathrm{m}^{2}$ (blue curves), and $\gamma=0.07 \mathrm{~J} / \mathrm{m}^{2}$ (red curves). As predicted by the theories, there is a marginal influence of the fractal dimension on the true contact area at small loads under loading conditions. The influence of $D_{f}$ becomes important during unloading. This leads to the formation of a hysteresis loading-unloading loop, which is strongly affected by the adhesion energy $\gamma$.

FIG. 7. The dimensionless load $\tilde{\sigma}=2 \sigma /\left(E^{*} q_{0} h_{\max }\right)$ (a) and the quantity $A / A_{0}$ (b) as a function of the nondimensional penetration $\tilde{\Delta}$, for two different Hurst exponents $H=$ $0.8\left(m_{2}=2.7 \times 10^{-3}\right), H=0.9\left(m_{2}=1.5 \times 10^{-3}\right)$, and for three different values of energy of adhesion, $\gamma=0.01 \mathrm{~J} / \mathrm{m}^{2}$ (black curves), $\gamma=0.04 \mathrm{~J} / \mathrm{m}^{2}$ (blue curves), and $\gamma=$ $0.07 \mathrm{~J} / \mathrm{m}^{2}$ (red curves). 